\title{
ABCG2 regulates the pattern of self-renewing divisions in cisplatin-resistant non-small cell lung cancer cell lines
}

\author{
YING TANG $^{1}$, JINGMING HOU ${ }^{2}$, GUANGHUI LI ${ }^{1}$, ZONGCHANG SONG ${ }^{1}$, \\ XIAOJING LI ${ }^{1}$, CUI YANG ${ }^{1}$, WENYING LIU ${ }^{1}$, YIDE HU ${ }^{1}$ and YU XU ${ }^{3}$ \\ ${ }^{1}$ Department of Oncology, Xinqiao Hospital, Third Military Medical University, Chongqing 400038; \\ ${ }^{2}$ Department of Orthopedics, Chinese PLA Beijing Army General Hospital, Beijing 100700; \\ ${ }^{3}$ Institute of Respiratory Disease, Xinqiao Hospital, Third Military Medical University, Chongqing 400038, P.R. China
}

Received July 15, 2014; Accepted August 22, 2014

DOI: $10.3892 /$ or.2014.3470

\begin{abstract}
Overexpression of ABCG2 is considered a major mechanism of cancer drug resistance. Recent studies have shown that $\mathrm{ABCG} 2$ can regulate the switch between symmetric and asymmetric cell division in adult stem cells; however, the relationship between ABCG2 and cell division in drugresistant cancer cells remains to be determined. In the present study, we demonstrated that ABCG2 is involved in the cell division of drug-resistant cancer cells. We first established drug-resistant H460 and A549 cell lines by repeated exposure to cisplatin and found that the expression of ABCG2 in these cell lines was significantly increased. As evidenced by PKH-26 staining, these drug-resistant cell lines favored symmetric division, which differed from the asymmetric division of the parental cells. Furthermore, we established stable ABCG2-overexpressing and stable shRNA-ABCG2-knockdown cell lines to evaluate the potential role of ABCG2 in cancer cell division. The results showed that overexpression of ABCG2 in A549 parental cells significantly increased the proportion of symmetric division, whereas knockdown of ABCG2 in drug-resistant A549 cells significantly increased the proportion of asymmetric division. Taken together, our findings suggest that ABCG2 is involved in the modulation of cancer drug resistance by regulating the pattern of cell division. The present study provides novel insight into the role of ABCG2 in cancer treatment resistance.
\end{abstract}

Correspondence to: Professor Yide Hu, Department of Oncology, Xinqiao Hospital, Third Military Medical University, 183 Xinqiao Street, Chongqing 400038, P.R. China

E-mail: huyide_mit@aliyun.com

Dr Yu Xu, Institute of Respiratory Disease, Xinqiao Hospital, Third Military Medical University, 183 Xinqiao Street, Chongqing 400038, P.R. China

E-mail: cqxuyu1982@qq.com

Key words: ABCG2, drug resistance, NSCLC, symmetric division, asymmetric division

\section{Introduction}

Chemotherapy is a key method of treatment in the primary and palliative care of patients with lung cancer, of which non-small cell lung cancer (NSCLC) accounts for the majority of cases. Cisplatin is one of the most common chemotherapeutic drugs for lung cancer treatment, particularly for NSCLC; however, many patients have resistance to cisplatin initially and secondarily $(1,2)$. To overcome drug resistance, patients with NSCLC are administered large doses of drugs, which induce numerous adverse effects and fail to improve the clinical prognosis. Therefore, a better understanding of the molecular mechanisms underlying cisplatin resistance is warranted to further clarify the exact mechanisms underlying chemoresistance and to find or design efficient drugs to improve individual chemotherapy strategies for NSCLC patients.

Increasing studies have shown that the active effluence of chemotherapeutic drugs from cancer cells is one of the main mechanisms of drug resistance. Cancer cells often exhibit drug resistance with the overexpression of membrane transport proteins, which effectively pump antitumor drugs out (3). The ATP-binding cassette (ABC) multi-drug transporters, such as ABCG2 (BCRP/MXR/ABCP), are considered to be responsible for the bulk of drug efflux in human cancer (4). Moreover, the overexpression of ABCG2 has been reported to confer drug resistance upon NSCLC to various chemotherapeutic drugs (5). Furthermore, a previous study also showed ABCG2 to be closely associated with clinical outcome in platinum-based chemotherapy for advanced NSCLC patients. For example, ABCG2-positive patients showed a lower response effect to chemotherapy and a shorter progression-free survival and low survival rate than ABCG2-negative patients (6).

However, the potential function of $\mathrm{ABCG} 2$ for cancer has not been completely elucidated. A number of recent reports have suggested that novel functions exist for this transporter besides drug efflux. A recent study demonstrated that ABCG2 was involved in the proliferation of cancer cells and suppression of ABCG2 inhibited cancer cell proliferation (10), which indicated that ABCG2 was involved in cancer cell proliferation. Notably, a recent study demonstrated that ABCG2 could directly regulate the conversion between symmetric and asymmetric cell division in a cardiac side population with 
determination of progenitor cell fate decisions (7). Although asymmetric cell division is a proposed characteristic of cancer stem cells, a recent study revealed that the majority of glioma stem cells were passaged through expansive symmetric cell division instead of asymmetric cell division (8). Moreover, there is emerging evidence that asymmetric division could function as a mechanism of tumor suppression in Drosophila neuroblasts. Loss-of-function mutations of cell polarity and cell fate determinants induce neuroblasts to divide symmetrically, leading to an increase in number, tissue overgrowth and transplantable tumors, which was ultimately similar to the generation of mammalian cancer (9). Whether or not there is a direct relationship between ABCG2 and cell division in drugresistant cancer cells remains to be determined. To date, the role of ABCG2 in cancer cell division remains unclear.

To explore the potential relationship between ABCG2 expression and the pattern of cancer cell division, we established cisplatin-resistant NSCLC cell lines and found that these cell lines have significantly increased expression of ABCG2. Markedly, as evidenced by PKH-26 staining, we found that these drug-resistant cell lines divided symmetrically more frequently than the parental cells. We therefore speculated that ABCG2 could regulate cell division in cisplatin-resistant NSCLC cells. To verify this hypothesis, we observed the cell division patterns of parental NSCLC cells that overexpressed ABCG2 and drug-resistant NSCLC cells with decreased ABCG2 expression by RNA interference. The result showed that ABCG2 regulated cell division in drug-resistant cancer cells.

\section{Materials and methods}

Cell lines and reagent. The human lung cancer cell lines, A549 and H460, were obtained from the American Type Culture Collection (ATCC) and routinely maintained in RPMI-1640 medium supplemented with $10 \%$ fetal bovine serum, $100 \mathrm{U} / \mathrm{ml}$ of penicillin, $100 \mathrm{mg} / \mathrm{ml}$ of streptomycin and $2 \mathrm{mM}$ L-glutamine. All cells were cultured as monolayer cultures and maintained in a humidified atmosphere of $5 \%$ $\mathrm{CO}_{2}$ in air at $37^{\circ} \mathrm{C}$. Cisplatin, 3-(4,5-dimethylthiazol-2-yl)2,5-diphenyltetrazolium bromide (MTT), propidium iodide (PI) and RNase A were purchased from Sigma-Aldrich Chemical Company (St. Louis, MO, USA). Antibodies against ABCG2 were purchased from Abcam, Inc. (Cambridge, MA, USA).

Drug sensitivity assay (MTT). Cells $\left(3 \times 10^{3}\right)$ were seeded in 96 -well plates and allowed to adhere overnight at $37^{\circ} \mathrm{C}$. The attached cells were then exposed to various concentrations of cisplatin for $72 \mathrm{~h}$. The MTT reagent [5 mg/ml $(20 \mu \mathrm{l} /$ well)] was added to each well for $4 \mathrm{~h}$. Subsequently, the medium was discarded and the purple MTT formazan was dissolved by DMSO (100 $\mu \mathrm{l} /$ well). Then, the absorbance value was measured at $490 \mathrm{~nm}$ and the concentration of cisplatin that induced $50 \%$ inhibition $\left(\mathrm{IC}_{50}\right)$ was calculated by GraphPad Prism software (version 5; GraphPad Software, Inc., San Diego, CA, USA).

Generation of drug-resistant cell lines. A549 and H460 cisplatin-resistant cell lines were derived from each original parental cell line by exposure to cisplatin following the $\mathrm{IC}_{50}$ value for $72 \mathrm{~h}$, and were then designated cisplatin-resistant (CisR) cells. The medium was removed and cells were cultured for an additional $72 \mathrm{~h}$ in normal medium. Cells were cultured with or without media containing cisplatin for $72 \mathrm{~h}$, repeatedly. This cycle was carried out for $\sim 6$ months, after which time the $\mathrm{IC}_{50}$ concentrations were re-assessed in each resistant cell line.

Establishment of stable ABCG2-overexpressing or shRNAABCG2 knockdown cell lines. A549 cells were transfected with the recombinant lentivirus of $\mathrm{ABCG} 2$ or control lentivirus (GFP-lentivirus), which was constructed by GeneChem Co. Ltd. (Shanghai, China). To establish A549/CisRshABCG2 cells, A549/CisR cells were transfected with SMART vector shRNA lentiviral particles targeted against the ABCG2 gene with $5 \mu \mathrm{g} / \mathrm{ml}$ of Polybrene (Sigma-Aldrich) to inhibit ABCG2 gene expression. An empty SMART vector expressing TurboGFP was used as control. The stable shRNA-ABCG2 cell line, A549/CisRshABCG2, was established and the expression of ABCG2 was evaluated by western blotting and PCR.

Western blot analysis. For western blot analysis, $40 \mu \mathrm{g}$ of total protein was separated by sodium dodecyl sulphate-polyacrylamide gel electrophoresis (SDS-PAGE), and the proteins were then transferred electrophoretically onto a polyvinylidene difluoride membrane. After blocking with 5\% skimmed milk in TBST buffer for $1 \mathrm{~h}$ at $37^{\circ} \mathrm{C}$, the membranes were incubated overnight at $4^{\circ} \mathrm{C}$ with specific primary antibody (rat antiBRCP/ABCG2 antibody, 1:500 dilution; Abcam). The protein levels were normalized against $\beta$-actin from the same sample (1:3,000 dilution; Boster, China). For detection, the membrane was incubated with goat anti-rat IgG antibody $(1: 10,000)$ in TBST for $1 \mathrm{~h}$ at room temperature. The immunoblots were imaged by an enhanced chemiluminescence (ECL) detection system, followed by exposure to ECL Hyperfilm (Beyotime, China).

Reverse transcription and quantitative real-time PCR assay. Total RNA was extracted from cells using TRIzol reagent (Invitrogen) following the manufacturer's instructions. The mRNAs were reverse-transcribed into cDNA using a PrimeScript $^{\mathrm{TM}}$ RT reagent kit (Takara Bio, Shiga, Japan). Then, a real-time quantitative PCR (RT-qPCR) assay was performed using the Applied Biosystems 7500 Sequence Detection system (Applied Biosystems, Foster City, CA, USA), and SYBR ${ }^{\circledR}$ Premix Ex Taq $^{\mathrm{TM}}$ II (Takara Bio). The primer sequences used in real-time RT-PCR were: ABCG2 forward, 5'-GAAACCTGGTCTCAACGCCATCC-3' and reverse, 5'-CGTCAGAGTGCCCATCACAACAT-3'; $\beta$-actin forward, 5'-CCTGGCACCCAGCACAAT-3' and reverse, 5'-GCC GATCCACACGGAGTACT-3'. The threshold cycle $(\mathrm{Ct})$ was used to determine the relative level of expression of each gene by normalizing to the $\mathrm{Ct}$ of $\beta$-actin. All genes were tested in 3 independent experiments.

PKH-26 staining. The cells from each group were washed with PBS and resuspended in PKH diluents with PKH dye labeling. The cells were incubated at room temperature for $5 \mathrm{~min}$ with periodic mixing. To stop the reaction, $2 \mathrm{ml}$ of serum was added, and the cells were incubated for $1 \mathrm{~min}$ to allow binding of excess dye. The samples were centrifuged and washed twice 

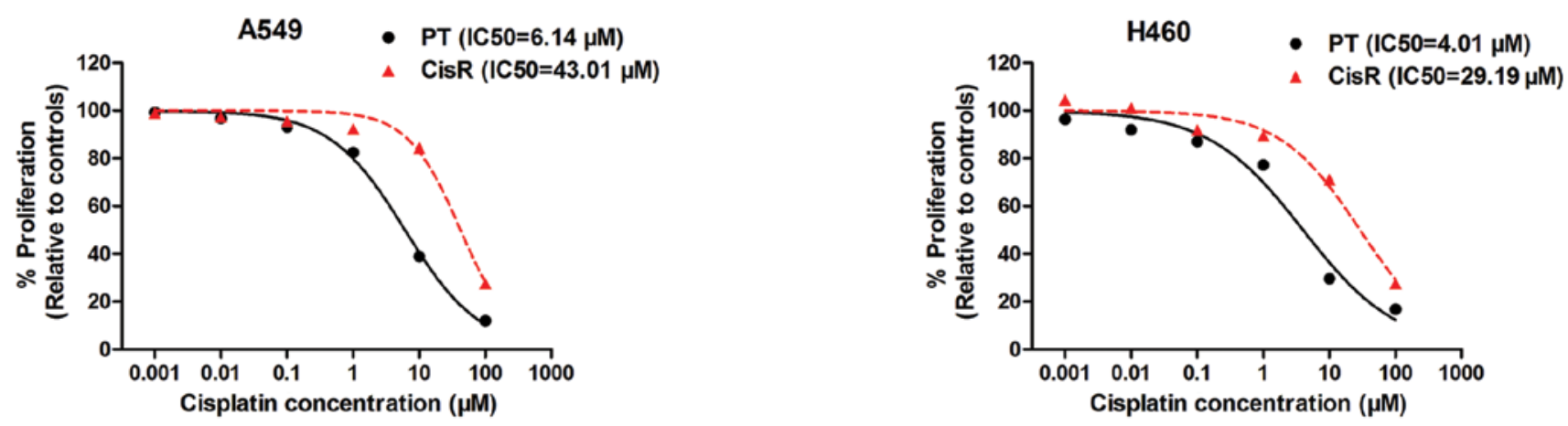

Figure 1. The $\mathrm{IC}_{50}$ concentrations detected in $\mathrm{A} 549$ and $\mathrm{H} 460$ cisplatin-resistant cell lines and the parental cell lines.

with $10 \mathrm{ml}$ of complete medium to remove the unbound dye. Finally, the cells were resuspended in DMEM serum-free medium supplemented with $20 \mathrm{ng} / \mathrm{ml}$ of EGF, $20 \mathrm{ng} / \mathrm{ml}$ of bFGF and $4 \mu \mathrm{g} / \mathrm{ml}$ of insulin, and plated on to ultra-low attachment 96-well at a density of 1,000/ml. PKH-26-stained cells were observed with fluorescence microscopy and images were acquired with an Olympus camera or confocal microscopy.

Statistical analysis. All data are presented as the means \pm standard deviation (SD) of at least 3 independent experiments. Analysis of variance (ANOVA) and two-tailed Student's t-tests were used to identify significant differences in the growth of sorted cells. Differences were considered to indicate a statistically significant result when the P-value was $<0.05$.

\section{Results}

Establishment and identification of cisplatin-resistant NSCLC cell lines. To determine $\mathrm{IC}_{50}$ values, cells were incubated with concentrations of cisplatin ranging from 0.001 to $100 \mu \mathrm{M}$. Dose-response curves were generated and $\mathrm{IC}_{50}$ concentrations were calculated (Fig. 1). Then, we established two cisplatinresistant lung cancer cell lines (H460/CisR and A549/CisR) by repeatedly treating parental $\mathrm{H} 460$ and A549 cells with the $\mathrm{IC}_{50}$ concentration of cisplatin. At 6 months, the $\mathrm{IC}_{50}$ values of H460/CisR and A549/CisR were also measured and calculated. The $\mathrm{IC}_{50}$ concentration of cisplatin-resistant cells displayed a significant increase compared to the corresponding parental cells (Fig. 1). In A549 cells, the $\mathrm{IC}_{50}$ concentration of cisplatinresistant cells was determined to be $43.01 \mu \mathrm{M}$ compared to $6.14 \mu \mathrm{M}$ in the original parental cell line; a 7-fold increase in the concentration of cisplatin was required to obtain a $50 \%$ inhibition in cell growth. A significant increase in $\mathrm{IC}_{50}$ concentrations was also observed in H460/CisR cells (29.19 vs. $4.01 \mu \mathrm{M}$ ), indicating a 7.28-fold increase in the $\mathrm{H} 460 /$ CisR cell lines compared to the parental cells. Taken together, the results demonstrated a cisplatin-resistant phenotype in two NSCLC cell lines following continuous exposure to cisplatin in vitro.

Overexpression of ABCG2 in cisplatin-resistant NSCLC cells. To observe the difference of the cisplatin-resistant cells and parental cells, we determined the level of expression of ABCG2 in A549, A549/CisR, H460 and H460/CisR cells by western blotting and real-time quantification PCR. The level of expression of ABCG2 in the A549/CisR cells was higher

\section{A Western blot}
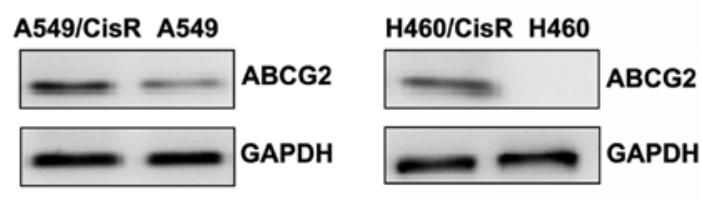

B RT-PCR

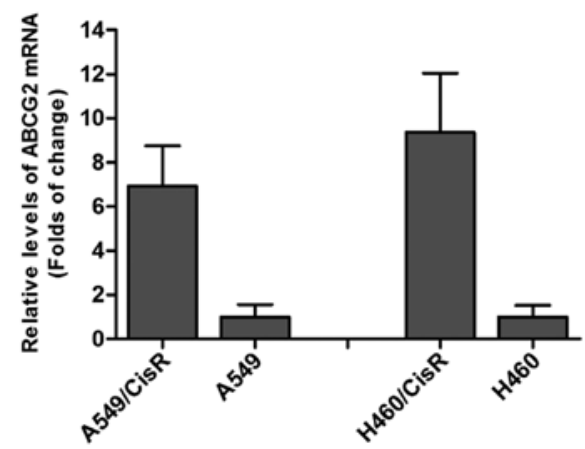

Figure 2. Levels of protein and mRNA expression of ABCG2 in cisplatinresistant NSCLC cells (A549/CisR and H460/CisR cells) and the parental cells (A549 and H460 cells). (A) Level of ABCG2 protein expression. (B) Level of ABCG2 mRNA expression. Fold-change of BCRP/ABCG2 mRNA expression was quantified relative to the internal control, $\beta$-actin.

than in the parental cells. Similar results were also observed in H460/CisR cells (Fig. 2). Moreover, a higher level of expression of ABCG2 mRNA was observed in A549/CisR cells and H460/CisR cells as compared to the parental cells (Fig. 2). The relative level of expression of ABCG2 mRNA in A549/CisR and H460/CisR cells was 6.92- or 9.36-fold higher than the corresponding parental cells, respectively. These results are consistent with numerous other studies, which demonstrated that ABCG2 had increased expression in human cancer cell lines screened by various anticancer drugs $(11,12)$.

Increased symmetric division in cisplatin-resistant NSCLC cells. To determine the pattern of cell self-renewing divisions in cisplatin-resistant cells (A549/CisR and H460/CisR cells) and the parental cells (A549 and H460 cells), we stained the four cell lines with PKH-26. PKH-26 is a fluorescent dye that binds to cell membranes and segregates in daughter cells after each cell division. This method is commonly used to detect the pattern of cell division (13). The results showed that the 

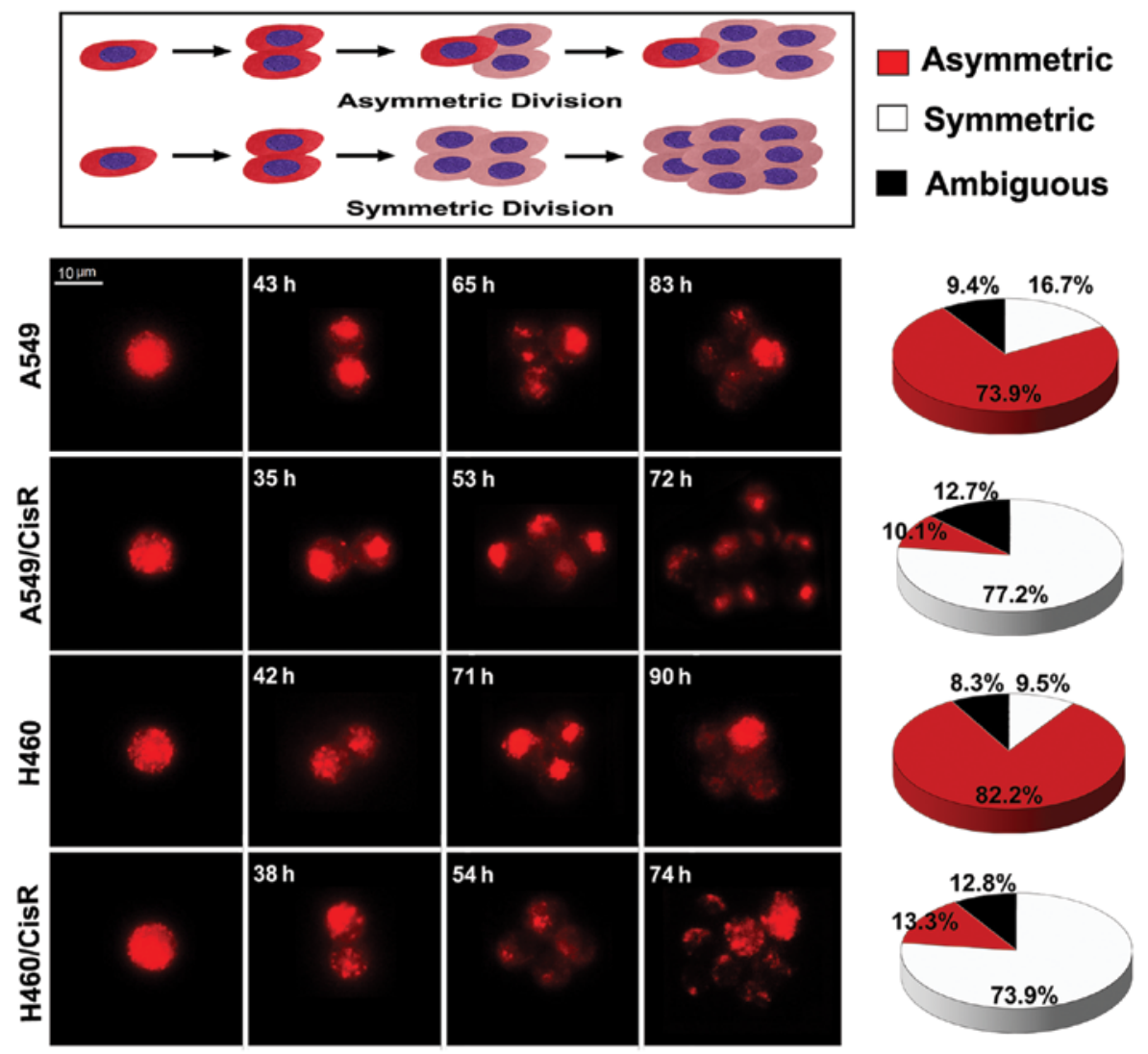

Figure 3. Proportions of asymmetric and symmetric divisions in cisplatin-resistant NSCLC cells (A549/CisR and H460/CisR cells) and the parental cells (A549 and $\mathrm{H} 460$ cells). The pie charts show the relative proportions of asymmetric and symmetric divisions.

pattern of cell division in A549 parental cells was $73.9 \%$ asymmetric, $16.7 \%$ symmetric, and $9.4 \%$ undefined (according to PHK-26 staining which could not be classified by PKH-26 dye). The pattern of cell division in $\mathrm{H} 460$ parental cells was similar to the pattern of A549 parental cells, including $82.2 \%$ asymmetric, $9.5 \%$ symmetric and $8.3 \%$ undefined. However, the patterns of cell division in cisplatin-resistant NSCLC cells were significantly different from the pattern of NSCLC parental cells (Fig. 3). The pattern of cell division in A549/CisR cells was $77.2 \%$ symmetric, $10.1 \%$ asymmetric and $12.7 \%$ undefined. The pattern of cell division in $\mathrm{H} 460 /$ CisR cells comprised of $73.9 \%$ symmetric, $13.3 \%$ asymmetric and $12.8 \%$ undefined. In summary, these results suggest that symmetric and asymmetric divisions co-exist in cisplatinresistant NSCLC cells and the parental cells, but with different proportions. Thus, parental NSCLC cells mainly divide asymmetrically, whereas cisplatin-resistant NSCLC cells mainly divide symmetrically.

Increased symmetric division in ABCG2-overexpressing NSCLC cell lines. The experiments described above indicated that cisplatin-resistant cells have increased symmetric division and display a higher level of ABCG2 expression. We next hypothesized that ABCG2 is involved in cell division in drug-resistant cells. To determine the relationship between ABCG2 and cell division in cisplatin-resistant NSCLC cells, we transfected the recombinant lentivirus of ABCG2 into A549 cells to establish a stable ABCG2-overexpressing cell line (A549/ABCG2). As shown in Fig. 4, the results of

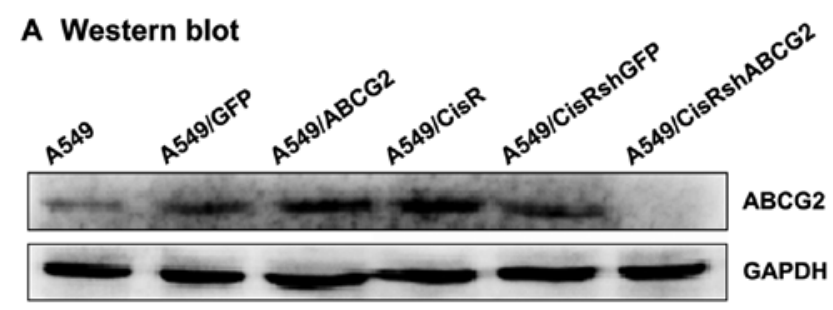

B RT-PCR

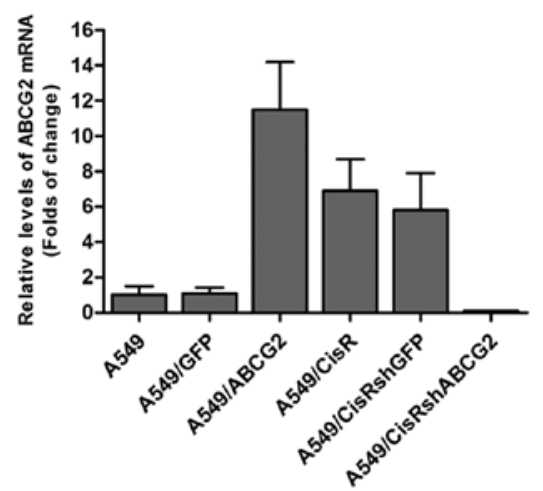

Figure 4. Levels of protein and mRNA expression of ABCG2 in ABCG2overexpressing stable cell lines and shRNA-mediated ABCG2 knockdown stable cell lines. (A) Level of ABCG2 protein expression. (B) Level of ABCG2 mRNA expression.

western blotting and real-time quantification PCR showed that the expression of ABCG2 in A549/ABCG2 cells was clearly increased. Moreover, PKH-26 staining showed that a 


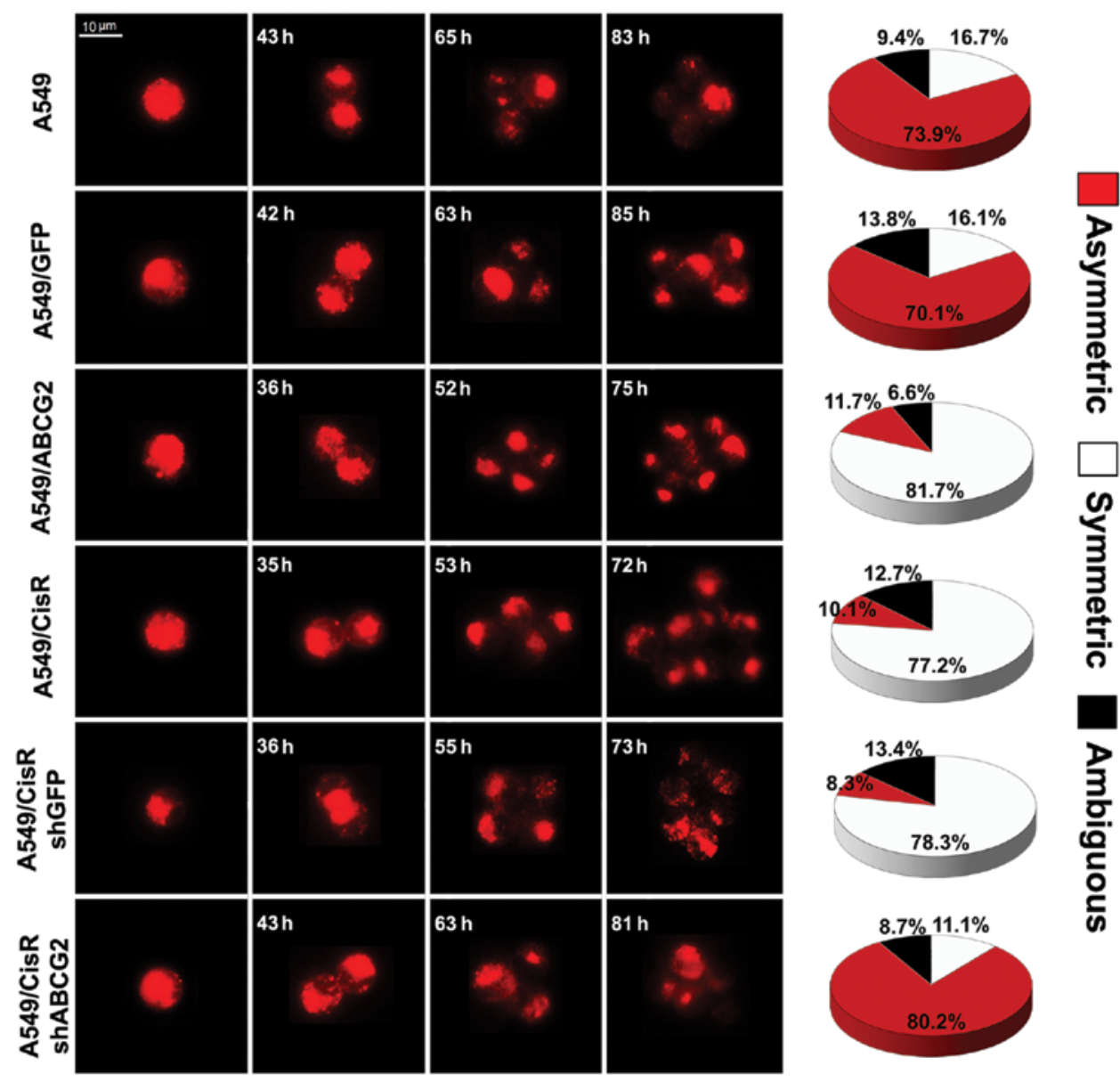

Figure 5. Proportions of asymmetric and symmetric division in ABCG2-overexpressing stable cell lines and shRNA-mediated ABCG2 knockdown stable cell lines. The charts show the distribution of cell division patterns.

significantly greater proportion of A549/ABCG2 cells (81.7\%) divided symmetrically than A549 parental cells (16.7\%; Fig. 5). These data indicate that ABCG2 overexpression increases the potential for symmetric division in A549 cells.

Increased asymmetric division in the ABCG2-inhibition cisplatin-resistant NSCLC cell line. To further confirm that the characteristics of ABCG2 described above could regulate cell division in NSCLC cells, we transfected A549/CisR cells with SMART vector shRNA lentiviral particles targeted against ABCG2 to inhibit expression of the gene. As shown in Fig. 4, the level of expression of ABCG2 in A549/CisRshABCG2 cells was significantly lower than A549/CisR cells. Furthermore, PKH-26 staining showed that a significantly lower percentage of A549/CisRshABCG2 cells (11.1\%) divided symmetrically than A549/CisR cells (77.2\%; Fig. 5). These findings, in addition to the observation that ABCG2 overexpression increased the percentage of symmetric division, indicate that ABCG2 regulates the pattern of cell self-renewing divisions in cisplatin-resistant NSCLC cell lines.

\section{Discussion}

ABCG2 is widely expressed in various normal tissues and stem cells, as well as cancer cells. Previous studies have suggested that $\mathrm{ABCG} 2$ plays a critical role in the maintenance of the stem cell phenotype and multidrug resistance of cancer cells $(14,15)$. However, to date, there have been no studies reporting the role of ABCG2 expression in cancer cell division. Hence, in the present study, we attempted to determine the possible role of ABCG2 in cell division in cisplatin-resistant NSCLC cells. Our data showed that $\mathrm{ABCG} 2$ is overexpressed in cisplatin-resistant NSCLC cells, and those cells were more prone to divide symmetrically, while silencing of ABCG2 in cisplatin-resistant NSCLC cells led to increased asymmetric division. Our study demonstrated that $\mathrm{ABCG} 2$ could regulate the pattern of cell division in cisplatin-resistant NSCLC cell lines.

Platinum-based chemotherapeutics, such as cisplatin, remain the standard first-line chemotherapy for NSCLC with good performance status and have shown significant improvement in overall survival and quality of life. However, cisplatin induces a variety of problems, such as cisplatin resistance, which is a major obstacle for successful cancer treatment (16). Furthermore, cisplatin-resistant tumors also fail to respond to other drugs (17). Thus, it is critical to gain a better understanding of the molecular mechanisms underlying the drug-resistant phenotype associated with cisplatin resistance. In the present study, we generated a clinically-relevant, isogenic model of cisplatin resistance in a panel of NSCLC cell lines from original, age-matched parent cell lines, and characterized these cell lines in terms of the fold-changes in $\mathrm{IC}_{50}$ values. Using $\mathrm{IC}_{50}$ concentrations, cisplatin-resistant cell lines 
were established over time through chronic in vitro exposure to cisplatin, after which time the $\mathrm{IC}_{50}$ values were re-assessed in cisplatin-treated cell lines. The $\mathrm{IC}_{50}$ of cisplatin-treated cells was shown to be significantly higher, demonstrating a more resistant phenotype of these cells. Although the exact mechanisms underlying cisplatin resistance are unclear, multidrug resistance has emerged as a significant cellular mechanism to explain clinical drug resistance and ABCG2 has been implicated in multidrug resistance in cancer chemotherapy (18). ABCG2 is a member of the ABC family and functions as an ATP-binding cassette discharge pump. ABCG2 prevents the intracellular accumulation of substrate compounds, including anticancer drugs, by limiting the influx into and facilitating the efflux out of cells. In cancer cells, high expression of ABCG2 prevents the intracellular accumulation of anticancer drugs, which results in drug resistance (19). In NSCLC, several studies have demonstrated that there is an association between high ABCG2 expression with platinum-based regimens and lower response rate, shorter overall survival and progressionfree survival $(6,20)$. In agreement with previous findings, our western blotting and PCR results showed that the level of ABCG2 expression in the cisplatin-resistant NSCLC cells was significantly higher than in the parental cells.

In the present study, our data also showed that the pattern of cell division in cisplatin-resistant NSCLC cells was significantly different from the pattern of cell division in the parental cells. Traditionally, there have been two basic models of stem cell division; asymmetric and symmetric (21). We found that symmetric and asymmetric division co-exist in cisplatin-resistant NSCLC and parental cells, but in different proportions; in particular, cisplatin-resistant NSCLC cells mainly divide symmetrically, whereas the parental cells mainly divide asymmetrically. According to an asymmetric cell division model, two daughter cells with divergent fates are generated, with one daughter cell capable of self-renewal and the other daughter cell committed to differentiation. According to a symmetric division model, two identical daughter cells are formed, which indicates that the cells retain stem cell properties or become committed cells early in the developmental process (22). The asymmetric model has the advantage of keeping the stem cell population level stable; however, an obvious disadvantage is an inability to replenish the stem cell pool in case of injury. This problem is naturally solved by the symmetric model (23). Several studies have shown that progenitor/stem cells divide asymmetrically during physiologic tissue homeostasis; however, these progenitor/stem cells favor symmetric division and rapid proliferation after tissue injury $(21,24)$. In addition, previous studies have shown that asymmetric division functions as a mechanism of tumor suppression in Drosophila neuroblasts. Loss-of-function mutations of cell polarity and cell fate determinants induce neuroblasts to divide symmetrically, leading to an increase in number, tissue overgrowth and ultimately transplantable tumors that resemble mammalian cancers (9). Recent evidence has revealed that cisplatin-resistant subpopulations of NSCLC cells have a putative stem-like character, including increased invasive ability and tumorigenic ability (25). Thus, our findings are consistent with previous studies, which showed an increased proportion of symmetric division in cisplatin-resistant NSCLC cells compared to the parental cells.
The most notable finding of the present study was the potential relationship between the level of ABCG2 expression and the pattern of NSCLC cell division. To better explain the molecular mechanisms involved in ABCG2-mediated cell division, we established stable ABCG2-overexpressing and ABCG2-knockdown cell lines and assessed cell division by $\mathrm{PKH}-26$ staining. We found that self-renewing division of ABCG2-overexpressing cells was more prone to divide symmetrically than the parental cells, and knockdown of ABCG2 expression could decrease the proportion of symmetric division in cisplatin-resistant A549 cells. These data provide evidence that ABCG2 can regulate the pattern of cell division in cisplatin-resistant NSCLC cell lines. These results were consistent with a recent study, which demonstrated that ABCG2 can directly regulate the switch between symmetric and asymmetric cell division in a cardiac side population (7).

Although our data showed that ABCG2 can regulate cell division in cisplatin-resistant NSCLC cells, the specific pathway is at present unknown. Given that ABCG2 is capable of transporting a diverse array of substrates, ABCG2 could participate in regulating cell division via the transport of exogenous or endogenous signaling molecules, which in turn may promote cell symmetric division. This hypothesis has been confirmed by several previous studies and some potential regulating pathways have been reported. For example, Susanto et al (26) showed that ABCG2 can regulate embryonic stem cell self-renewal through maintenance of porphyrin homeostasis. Cicalese et al (27) suggested that p53 regulated polarity of cell division in mammary cancer stem cells and loss of p53 favored symmetric divisions of cancer cells, thus contributing to tumor growth. Additionally, an increased expression of p53 has been reported in ABCG2 knockout mice (7). Further studies are needed to clarify the mechanism by which ABCG2 regulates cancer cell division.

Moreover, the present study may provide a new strategy for the clinical treatment of NSCLC. Chemotherapy is typically administered in cycles with 3 -week intervals to recover the hematopoietic system and other normal cells. However, previous studies have reported that tumor cells can aggressively repopulate during these intervals and implant into the pre-treated location (28). We hypothesized that conventional chemotherapy can increase the level of expression of ABCG2, which further increases the proportion of symmetric division in NSCLC cells and promotes rapid cancer proliferation and restores the tumor to its pre-treatment size. This hypothesis has been supported by several recent studies. For example, Chen et al (10) reported that ABCG2 is involved in the proliferation of cancer cells. Cicalese et al (27) demonstrated that loss of p53 promotes symmetric division and contributes to tumor rapid growth, and pharmacologic reactivation of $\mathrm{p} 53$ is correlated with restoration of asymmetric divisions and tumor growth reduction. Collectively, our findings together with previous reports, suggest that therapies aimed to increase asymmetric division inhibit the rapid proliferation of NSCLC cells, and potentially improve long-term outcomes for lung cancer patients.

The decreased accumulation of antitumor drugs mediated by ABCG2 is considered to be one of the cellular mechanisms involved in drug resistance of cancer cells. In the present study, we found that ABCG2 also contributes to symmetric 
division in cancer cells, particularly in drug-resistant cancer cells. Therefore, ABCG2 is involved in the modulation of drug resistance through regulation of the pattern of cell division and drug effluence. Our findings provide a better understanding of the function of ABCG2 in cancer chemoresistance; however, additional research is required to study the new function of this transporter and to explain the interactions between ABCG2 and pathways that regulate the cell division of stem and cancer cells.

\section{Acknowledgements}

This study was supported by the National Natural Science Foundation of China (grant no. 81201684).

\section{References}

1. Bunn PA Jr and Kelly K: New chemotherapeutic agents prolong survival and improve quality of life in non-small cell lung cancer: a review of the literature and future directions. Clin Cancer Res 4: 1087-1100, 1998.

2. Giaccone G, Splinter TA, Debruyne C, et al: Randomized study of paclitaxel-cisplatin versus cisplatin-teniposide in patients with advanced non-small-cell lung cancer. The European Organization for Research and Treatment of Cancer Lung Cancer Cooperative Group. J Clin Oncol 16: 2133-2141, 1998.

3. Gottesman MM, Fojo T and Bates SE: Multidrug resistance in cancer: role of ATP-dependent transporters. Nat Rev Cancer 2: 48-58, 2002.

4. Robey RW, To KK, Polgar O, et al: ABCG2: a perspective. Adv Drug Deliv Rev 61: 3-13, 2009.

5. Nakamura Y, Oka M, Soda H, et al: Gefitinib ('Iressa', ZD1839), an epidermal growth factor receptor tyrosine kinase inhibitor, reverses breast cancer resistance protein/ABCG2-mediated drug resistance. Cancer Res 65: 1541-1546, 2005.

6. Yoh K, Ishii G, Yokose T, et al: Breast cancer resistance protein impacts clinical outcome in platinum-based chemotherapy for advanced non-small cell lung cancer. Clin Cancer Res 10 1691-1697, 2004.

7. Sereti KI, Oikonomopoulos A, Unno K, Cao X, Qiu Y and Liao R: ATP-binding cassette G-subfamily transporter 2 regulates cell cycle progression and asymmetric division in mouse cardiac side population progenitor cells. Circ Res 112: 27-34, 2013.

8. Lathia JD, Hitomi M, Gallagher J, et al: Distribution of CD133 reveals glioma stem cells self-renew through symmetric and asymmetric cell divisions. Cell Death Dis 2: e200, 2011.

9. Gonzalez C: Spindle orientation, asymmetric division and tumour suppression in Drosophila stem cells. Nat Rev Genet 8: 462-472, 2007

10. Chen Z, Liu F, Ren Q, et al: Suppression of ABCG2 inhibits cancer cell proliferation. Int J Cancer 126: 841-851, 2010.

11. To KK, Robey RW, Knutsen T, Zhan Z, Ried T and Bates SE: Escape from hsa-miR-519c enables drug-resistant cells to maintain high expression of ABCG2. Mol Cancer Ther 8: 2959-2968, 2009.
12. Litman T, Brangi M, Hudson E, et al: The multidrug-resistant phenotype associated with overexpression of the new $\mathrm{ABC}$ half-transporter, MXR (ABCG2). J Cell Sci 113: 2011-2021, 2000.

13. Lanzkron SM, Collector MI and Sharkis SJ: Hematopoietic stem cell tracking in vivo: a comparison of short-term and long-term repopulating cells. Blood 93: 1916-1921, 1999.

14. Doyle L and Ross DD: Multidrug resistance mediated by the breast cancer resistance protein BCRP (ABCG2). Oncogene 22: 7340-7358, 2003.

15. Ueda T, Brenner S, Malech HL, et al: Cloning and functional analysis of the rhesus macaque ABCG2 gene. Forced expression confers an SP phenotype among hematopoietic stem cell progeny in vivo. J Biol Chem 280: 991-998, 2005.

16. Rosell R, Lord RV, Taron M and Reguart N: DNA repair and cisplatin resistance in non-small-cell lung cancer. Lung Cancer 38: 217-227, 2002.

17. Ikuta K, Takemura K, Sasaki K, et al: Expression of multidrug resistance proteins and accumulation of cisplatin in human non-small cell lung cancer cells. Biol Pharm Bull 28: 707-712, 2005.

18. Xu J, Peng $\mathrm{H}$ and Zhang JT: Human multidrug transporter ABCG2, a target for sensitizing drug resistance in cancer chemotherapy. Curr Med Chem 14: 689-701, 2007.

19. Doyle LA, Yang W, Abruzzo LV, et al: A multidrug resistance transporter from human MCF-7 breast cancer cells. Proc Natl Acad Sci USA 95: 15665-15670, 1998.

20. Ota S, Ishii G, Goto $\mathrm{K}$, et al: Immunohistochemical expression of BCRP and ERCC1 in biopsy specimen predicts survival in advanced non-small-cell lung cancer treated with cisplatin-based chemotherapy. Lung Cancer 64: 98-104, 2009.

21. Morrison SJ and Kimble J: Asymmetric and symmetric stem-cell divisions in development and cancer. Nature 441: 1068-1074, 2006.

22. Knoblich JA: Mechanisms of asymmetric stem cell division. Cell 132: 583-597, 2008.

23. Shahriyari L and Komarova NL: Symmetric vs. asymmetric stem cell divisions: an adaptation against cancer? PLoS One 8: e76195, 2013.

24. Zhang R, Zhang Z, Zhang C, et al: Stroke transiently increases subventricular zone cell division from asymmetric to symmetric and increases neuronal differentiation in the adult rat. J Neurosci 24: 5810-5815, 2004.

25. Barr MP, Gray SG, Hoffmann AC, et al: Generation and characterisation of cisplatin-resistant non-small cell lung cancer cell lines displaying a stem-like signature. PLoS One 8: e54193, 2013.

26. Susanto J, Lin YH, Chen YN, et al: Porphyrin homeostasis maintained by ABCG2 regulates self-renewal of embryonic stem cells. PLoS One 3: e4023, 2008.

27. Cicalese A, Bonizzi G, Pasi CE, et al: The tumor suppressor $p 53$ regulates polarity of self-renewing divisions in mammary stem cells. Cell 138: 1083-1095, 2009.

28. Kim JJ and Tannock IF: Repopulation of cancer cells during therapy: an important cause of treatment failure. Nature Rev Cancer 5: 516-525, 2005. 